\title{
Improved fast-rotating black hole evolution simulations with modified Baumgarte-Shapiro-Shibata-Nakamura formulation
}

\author{
Hwei-Jang Yo, ${ }^{1}$ Zhoujian Cao, ${ }^{2}$ Chun-Yu Lin, ${ }^{3}$ and Hsing-Po Pan ${ }^{1}$ \\ ${ }^{1}$ Department of Physics, National Cheng-Kung University, Tainan 701, Taiwan \\ ${ }^{2}$ Institute of Applied Mathematics and LSEC, Academy of Mathematics and Systems Science, \\ Chinese Academy of Sciences, Beijing 100190, China \\ ${ }^{3}$ National Center for High-Performance Computing, Hsinchu 300, Taiwan
}

(Dated: January 18, 2021)

\begin{abstract}
Different formulations of Einstein's equations used in numerical relativity can affect not only the stability but also the accuracy of numerical simulations. In the original Baumgarte-Shapiro-ShibataNakamura (BSSN) formulation, the loss of the angular momentum, $J$, is non-negligible in highly spinning single black hole evolutions. This loss also appears, usually right after the merger, in highly spinning binary black hole simulations, The loss of $J$ may be attributed to some unclear numerical dissipation. Reducing unphysical dissipation is expected to result in more stable and accurate evolutions. In the previous work [1] we proposed several modifications which are able to prevent black hole evolutions from the unphysical dissipation, and the resulting simulations are more stable than in the traditional BSSN formulation. Specifically, these three modifications (M1, M2, and M3) enhance the effects of stability, hyperbolicity, and dissipation of the formulation. We experiment further in this work with these modifications, and demonstrate that these modifications improve the accuracy and also effectively suppress the loss of $J$, particularly in the black hole simulations with the initially large ratio of $J$ and the square of the ADM mass.
\end{abstract}

PACS numbers: 04.25.Dm, 04.30.Db, 95.30.Sf, 97.60.Lf

\section{INTRODUCTION}

Development of numerical relativity has been rapid after the breakthroughs in 2005 and 2006 (see, e.g., [2 4] ). Numerical relativity has now become an indispensable and effective tool in the research of general relativity and relativistic astrophysics. It has been extensively studied in several areas; and applied to the construction of gravitational waveform template banks for detection [5], to the kick phenomena of general binary systems [ 6 - 10], and to astrophysical problems such as the equation of state of neutron stars 11 14, electromagnetic counterparts of gravitational waves [15, 16], gamma ray bursts [17], accretion disks [18 and so on. These applications and numerical investigations demand increasingly greater accuracy, besides stability; thus it is important and necessary to unremittingly refine the formulations and schemes.

Among the methods to enhance the stability and accuracy in numerical relativity, the $3+1$ formulation of Einstein's equations is favored by many researchers. The Baumgarte-Shapiro-Shibata-Nakamura (BSSN) formulation [19, 20] is the most popular scheme, and it is usually implemented in first-order-in-time and second-orderin-space finite-differencing codes. Many works have been focused on modifying the original BSSN formulation to achieve better numerical stability and accuracy [21 26]. For example, borrowing from ideas in the Generalized Harmonic (GH) formulation [2, 27], the Z4 conformal (Z4c) formulation 28] and the traceless-conformal and covariant Z4 (CCZ4) formulation 29] both show good constraint damping behavior 30 32].

There is still room to improve the BSSN formulation to obtain better stability and accuracy, e.g., see references
21 23, 33 35]. In [1], we adopt a different approach from the CCZ4 and Z4c formulations to modify the BSSN formulation. The basic idea is to suppress the numerical error by adding combinations of constraint terms to the field equations in the BSSN formulation without changing the solution analytically, to modify the leading terms of the field equations. And we demonstrated that our modifications achieved more stable simulations than the traditional BSSN formulation. Specifically, our last work 1] has shown improvements in constraint damping and in the late-time behavior of the gravitational waveforms. In this work, we would like to emphasize the effectiveness of these modifications on the evolution of the black holes with higher spins, hoping to meet the demand of modeling extreme sources for the gravitational wave detection.

It was found in [36] that the angular momentum decays right after the final black hole forms in the binary black hole evolution simulations. For the single spinning black hole, the angular momentum also decays when the dimensionless spin $s / m^{2}>0.75$ (compare the cases $s / m^{2}=0.53$ and $s / m^{2}=0.9$ in Fig. 4 of [36]). This decay is neither due to the resolution nor the initial separation of the binary [36]. In contrast, the result from the SpEC code does not show this tendency [37]. For comparing those results, we plot in Fig. 1 $\chi_{f} \equiv s_{f} / m_{f}^{2}$ of the final black hole as a function of the initial spin parameter $\chi_{i} \equiv s_{i} / m_{i}^{2}$ for individual black hole component. We find that they are consistent when $\chi_{i}<0.75$ and, when $\chi_{i}$ getting larger, $\chi_{f}$ in the traditional BSSN formulation becomes smaller than that in the GH formulation. The difference should not be attributed to whether the spectral method or the finite-differencing method is used. However, it is yet unclear if the issue comes from the for- 


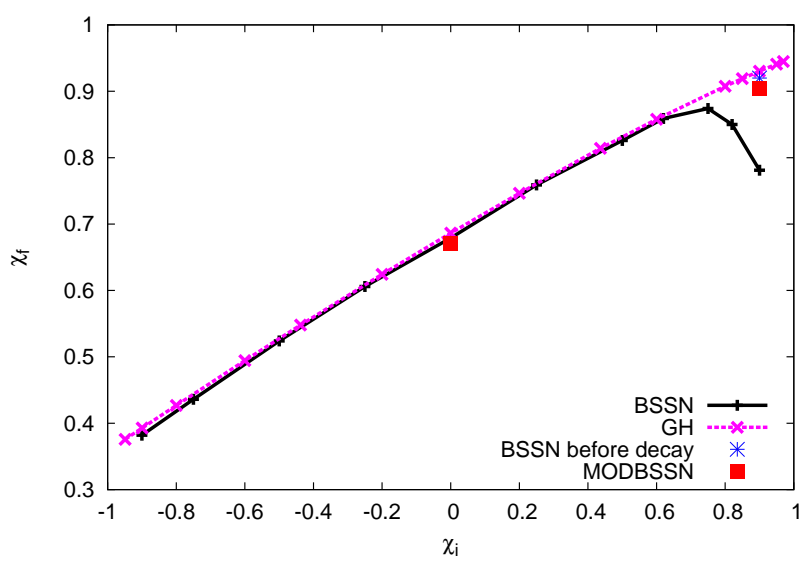

FIG. 1: Dimensionless spin parameter $\chi_{f}$ of the final black hole as a function of the initial dimensionless spin parameter $\chi_{i}$ of the individual black hole in the binary black hole evolution for the BSSN and GH formulations. The data 'BSSN' given in 36] are calculated through the BAM code with BSSN formulation and the finite-differencing method. The data 'GH' given in [37] are calculated through the SpEC code with GH formulation and the spectral method. The data 'BSSN before decay' given in [36] is calculated through the LEAN code 36]. It corresponds to the result right after the final black hole forms, and the data 'MODBSSN' are calculated through AMSS-NCKU code in current work, and the modified BSSN formulation (check the main text for more detail) is used.

mulation itself or from the puncture method. In this work, we therefore try to resolve this problem by simulating single and binary black hole evolutions with the modified BSSN formulation as proposed in [1]. We will show that the angular momentum is more accurate and its conservation is much better than in the traditional BSSN formulation. Via the better conservation of the angular momentum, it is also expected that the accuracy of the other related physical quantities will be improved at the same time with the modified formulation.

The rest of this work is organized as follows: In the next section, we will give an explicit description of the modifications to the BSSN formulation, discuss the related accuracy problems, and describe the numerical implementation. We then report on the test results on single spinning black hole in Sec. IIIA. The results for highly spinning binary black hole are presented in Sec. IIIB And the discussion and summary will be presented in the Sec. IV] Throughout the paper, geometric units with $G=c=1$ are used. Einstein summation rule is adopted unless stated explicitly.

\section{MODIFICATIONS AND NUMERICAL IMPLEMENTATIONS}

The BSSN formulation and the numerical recipes for implementation have been described in details in previ- ous articles 22, 34]. Here we only mention several major steps that have usually been adopted [21, 33] in the traditional BSSN formulation:

- In order to enforce the algebraic constraints of the unimodular determinant of $\tilde{\gamma}_{i j}$, i.e., $\tilde{\gamma}=1$, and of the tracelessness of $\tilde{A}_{i j}$, i.e., $\tilde{\gamma}^{i j} \tilde{A}_{i j}=0$, the numerical values of $\tilde{\gamma}_{i j}$ and $\tilde{A}_{i j}$ are replaced with $\tilde{\gamma}_{i j} \rightarrow \tilde{\gamma}^{-1 / 3} \tilde{\gamma}_{i j}, \tilde{A}_{i j} \rightarrow \tilde{A}_{\langle i j\rangle}$ after every time step, wherein the two indices in the angle bracket \langle\rangle is taken to be its symmetric and traceless part.

- The conformal connection functions $\tilde{\Gamma}^{i}$ are promoted to be independent variables in the BSSN formulation, which leads to the $\Gamma$-constraints $\mathcal{G}^{i} \equiv$ $\tilde{\Gamma}^{i}-\tilde{\Gamma}_{\mathbf{g}}^{i}=0$, where $\tilde{\Gamma}_{\mathbf{g}}^{i} \equiv \tilde{\gamma}^{j k} \tilde{\Gamma}^{i}{ }_{j k}$. The conventional approach to enforce the $\Gamma$-constraints is to replace all the undifferentiated $\tilde{\Gamma}^{i}$ with $\tilde{\Gamma}_{\mathbf{g}}^{i}$.

- The high-order Kreiss-Oliger (KO) method is employed to dissipate effectively the numerical noise.

These traditional approaches with suitable gauge condition have enabled fruitful studies on the black hole problem. Yet earlier investigations, e.g., 36], indicated that, in some near extreme situation, the traditional BSSN formulation is not robust enough to conserve the constraints and global quantities. We plan to test the following modifications which have been introduced in [1], and compare the results from our modifications with those from the traditional BSSN formulation. The three proposed modifications are as follows:

\section{A. Modification M1}

Instead of replacing all the undifferentiated $\tilde{\Gamma}^{i}$ with $\tilde{\Gamma}_{\mathbf{g}}^{i}$, M1 modifies the conformal 3-connection appearing in the right-hand-side of all the field equations, and changes the linear terms in the field equation of $\tilde{\Gamma}^{i}$. The new conformal 3 -connection in all field equations now takes the form

$$
\tilde{\Gamma}^{i}{ }_{j k} \rightarrow \tilde{\Gamma}^{i}{ }_{j k}-\frac{3}{5} \delta^{i}{ }_{\langle j} \tilde{T}_{k\rangle}-\frac{1}{5} \delta^{i}{ }_{\langle j} \mathcal{G}_{k\rangle}+\frac{1}{3} \tilde{\gamma}_{j k} \mathcal{G}^{i},
$$

where $\tilde{T}_{i} \equiv \tilde{\Gamma}^{k}{ }_{k i}=(\ln \sqrt{\tilde{\gamma}})_{, i}$ vanishes analytically, but could be nonzero due to numerical error. This expression is motivated by the unique algebraic decomposition for any third-rank symmetric tensor with two indices. See 1] for the details.

To change the behavior of the linear term in the field equation of $\tilde{\Gamma}^{i}$, we replace the original field equations of $\tilde{\Gamma}^{i}$ with

$$
\begin{aligned}
\partial_{t} \tilde{\Gamma}^{i}= & 2 \alpha\left[\tilde{\Gamma}^{i}{ }_{j k} \tilde{A}^{j k}-\frac{2}{3}\left(\tilde{\gamma}^{i j} K\right)_{, j}+6 \tilde{A}^{i j} \phi, j\right]-2 \tilde{A}^{i j} \alpha_{, j} \\
& +\beta^{j} \tilde{\Gamma}^{i}{ }_{, j}-\tilde{\Gamma}^{j} \beta^{i}{ }_{, j}+\tilde{\gamma}^{j k} \beta^{i}{ }_{, j k}+\frac{1}{3} \tilde{\gamma}^{i j} \beta^{k}{ }_{, j k} \\
& +\frac{2}{3}\left(\beta^{k}{ }_{, k}-2 \alpha K\right) \tilde{\Gamma}^{i}-(1+\xi) \Theta\left(\lambda^{i}\right) \lambda^{i} \mathcal{G}^{i},
\end{aligned}
$$


wherein $\Theta(x)$ is the step function

$$
\Theta(x)= \begin{cases}0 & \text { if } x<0 \\ 1 & \text { if } x>0\end{cases}
$$

and $\lambda^{i}$ is

$$
\lambda^{i}=\frac{2}{3}\left(\beta_{, k}^{k}-2 \alpha K\right)-\beta_{, \hat{i}}^{\hat{i}}-\frac{2}{5} \alpha \tilde{A}_{\hat{i}}^{\hat{i}} .
$$

Note that the index with hat, i.e., $\hat{i}$, means that no index summation is carried out with respect to this index. $\xi$ is chosen to be 1 in all cases in this work. This modification plays an indispensable role in the whole modification scheme to enhance both the stability and accuracy of the system.

Notice that there is one term in eqn (2) including a step function, i.e., $(1+\xi) \Theta\left(\lambda^{i}\right) \lambda^{i} \mathcal{G}^{i}$. Due to its switch character and the possible sign fluctuation of the numerical value of its argument $\lambda^{i}$ when $\lambda^{i}$ is close to zero, the step function should be sensitive to the resolution used in simulations. So we expect that this modification could affect majorly the numerical convergence behavior of the modified BSSN formulation.

\section{B. Modification M2}

The idea behind M2 is similar to that in obtaining Eq. (11). The algebraic structure of $\partial_{t} \tilde{\gamma}_{i j}$, similar to the algebraic structure of the conformal 3-connection, allows us to write the $\tilde{\gamma}_{i j}$-field equation as

$$
\partial_{t} \tilde{\gamma}_{i j} \rightarrow \partial_{t} \tilde{\gamma}_{i j}+\sigma \beta_{(i} \mathcal{G}_{j)}-\frac{1}{5} \tilde{\gamma}_{i j} \beta^{k} \mathcal{G}_{k}
$$

and we set $\sigma=1 / 10$ in this work. This modification enhances the hyperbolicity of the system and propagates the constraint violation residual away effectively.

\section{Modification M3}

This dissipation type of modification M3 is motivated from [23]. The major difference is that we use the symmetric traceless part of the partial derivative of the momentum constraint (instead of the symmetric part of its covariant derivative as in [23]) to re-write the $\tilde{A}_{i j}$-field equation as

$$
\partial_{t} \tilde{A}_{i j} \rightarrow \partial_{t} \tilde{A}_{i j}+h^{2} \mathcal{M}_{\langle i, j\rangle},
$$

wherein $\mathcal{M}_{i}$ is the momentum constraint, $h$ is the grid width. This modification provides a dissipation mechanism on $\tilde{A}_{i j}$, and serves as a natural alternative to the $\mathrm{KO}$ dissipation. In this work, we apply this modification instead of the $\mathrm{KO}$ method to check its capability in dissipation and also compare its effect with KO's.

There is a concern about the convergence of the whole system with this modification. At first glance, Equation
(6) might change the convergence order of a system to be only second-order accurate at most since the addtion term in the equation, i.e., $h^{2} \mathcal{M}_{\langle i, j\rangle}$, is only proportional explicitly to $h^{2}$. However, this is not the case. If one system is $p^{\text {th }}$-order convergent, then the momentum constraint $\mathcal{M}_{i} \simeq 0$ will converge to zero with the rate of $h^{p}$. So will the term $\mathcal{M}_{\langle i, j\rangle}$. Therefore, if we combine the convergence order of $\mathcal{M}_{\langle i, j\rangle}$ and the the multiplier $h^{2}$, the term introduced in eqn (6) will converge to zero with the rate $h^{p+2}$, which convergence is faster than the rest of the system. Thus this modification will not reduce the convergence order of the system analytically.

\section{Numerical Implementation}

The AMSS-NCKU code with the standard moving box style mesh refinement [1, 34, 38] is used in this work. We used 10 mesh levels, all of which are fixed in the cases of single black hole evolution, and the finest 3 levels are movable in evolving the binary black holes (BBHs). In each fixed level, we used one box with $128 \times 128 \times 64$ grids with assumed equatorial symmetry. The outermost physical boundary is $512 M$ and this makes the finest resolution to be $h=M / 64$. For the movable levels, two boxes with $64 \times 64 \times 32$ grids are used to cover each black hole. In time direction, the Berger-Oliger numerical scheme is adopted for the levels higher than four.

The moving puncture gauge condition

$$
\begin{aligned}
& \partial_{t} \alpha=\beta^{i} \alpha_{, i}-2 \alpha K, \\
& \partial_{t} \beta^{i}=\frac{3}{4} B^{i}+\beta^{j} \beta_{, j}^{i}, \\
& \partial_{t} B^{i}=\partial_{t} \tilde{\Gamma}^{i}-\eta B^{i}+\beta^{j} B_{, j}^{i}-\beta^{j} \tilde{\Gamma}_{, j}^{i} .
\end{aligned}
$$

is used and has been shown to give good behavior for the black hole simulations in [34]. In this paper we use $\eta=2 M$ with $M$ being the ADM mass of the given configuration.

\section{NUMERICAL RESULTS}

\section{A. Single black hole tests}

In this subsection, we test our modifications in spinning single black hole ( $\mathrm{SBH}$ ) cases with respective initial dimensionless spin parameters $\chi_{0} \equiv J_{0} / M_{0}^{2}=0.53$, 0.9 , and 0.923 . To generate these sets of puncture initial data with unit ADM mass, the bare mass and the spin parameter in the $z$-direction are set to be $m=0.872335$, 0.3528 , and 0.215898 and $s_{z}=0.53,0.45$, and 0.472466 respectively, as the input for the TwoPuncture solver. Note that $\chi_{0}=0.923$ is nearly the maximal spin that the conformally flat Bowen-York initial data can achieve [39]. The global quantities such as the ADM mass $M$ and the angular momentum $J$ are calculated with the surface integrals at $R=50 \mathrm{M}$, as described in [34]. 

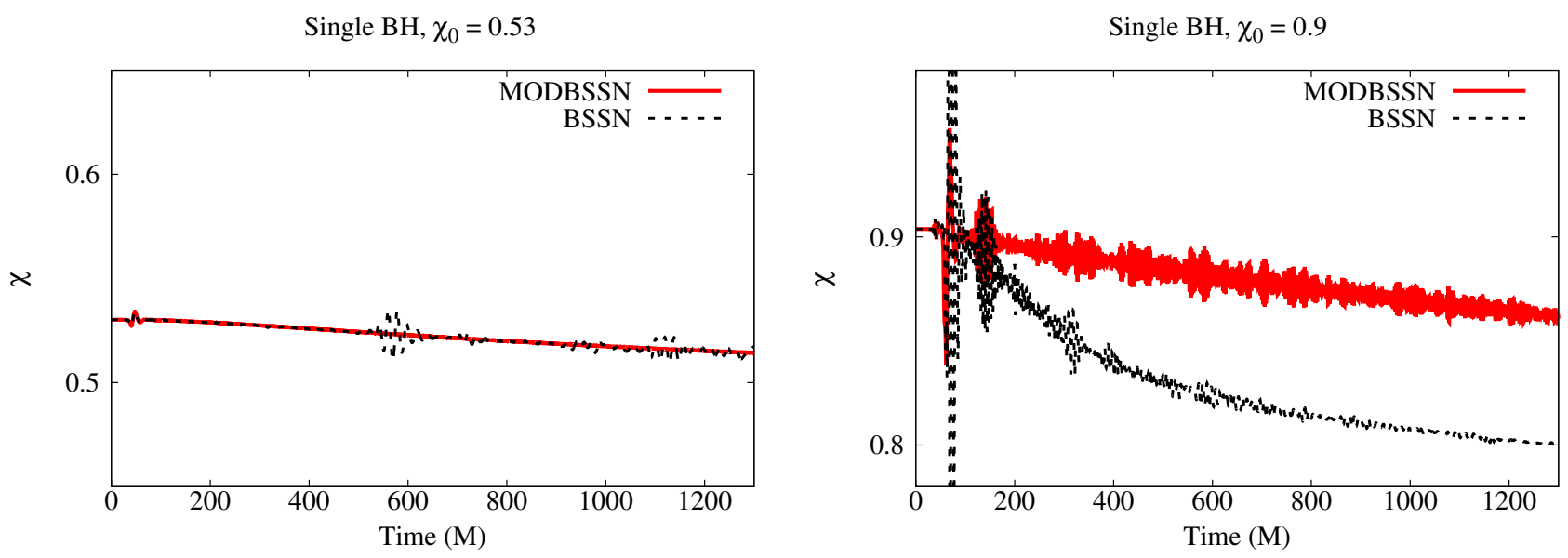

FIG. 2: Dimensionless spin parameter $\chi$ as a function of time for $\chi_{0}=0.53$ (left column) and $\chi_{0}=0.9$ (right column) in the single black hole evolutions. The modified BSSN formulation (solid red line, marked as MODBSSN) is shown to produce less noise in the spurious radiation as well as preserving $\chi$ better than the traditional BSSN formulation (dashed line, marked as BSSN) in the higher spin case.
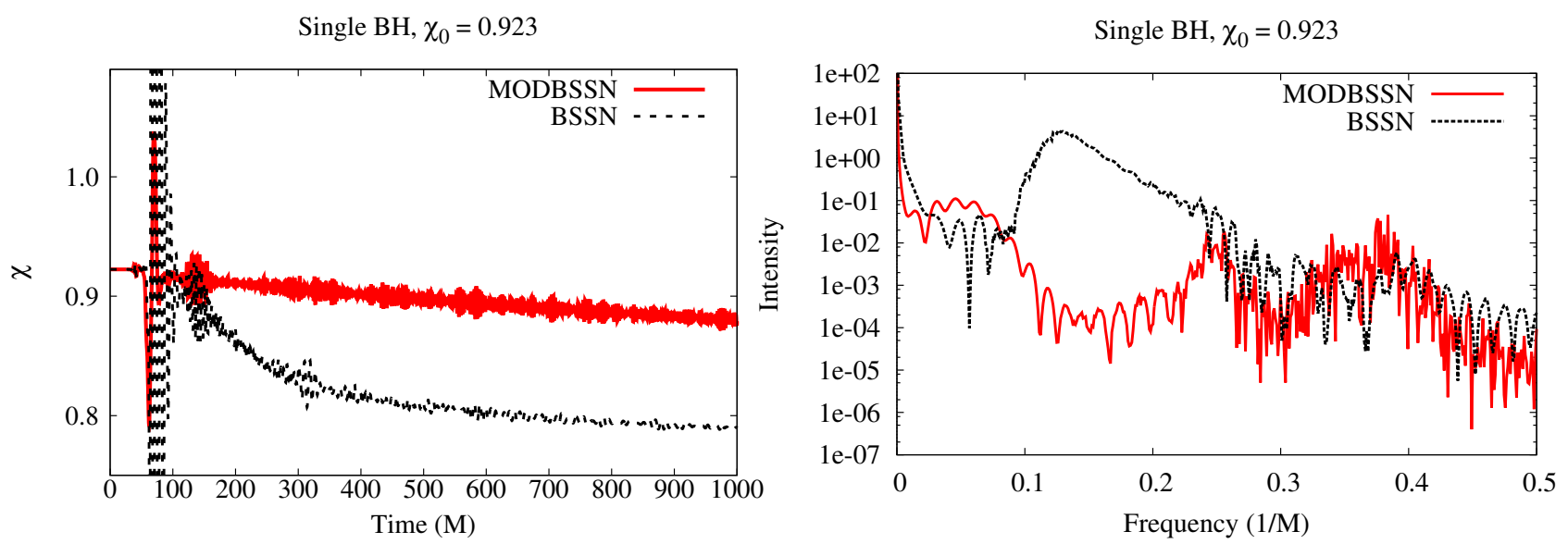

FIG. 3: Left: Dimensionless spin parameter $\chi$ as a function of time for $\chi_{0}=0.923$ in the single black hole evolution. The modified BSSN formulation (solid red line, marked as MODBSSN) is shown to preserve $\chi$ better than the traditional BSSN formulation (dashed line, marked as BSSN) in this near extreme case. Right: Power spectrum of the corresponding data plotted in the left panel.

The dimensionless spin parameter $\chi \equiv J / M^{2}$ for the single black hole simulation is shown in Fig. 2 and Fig. 3 . For the cases with the traditional BSSN formulation, it shows that the result is consistent with the Fig. 4 of [36]. It is also clear in the figures that the proposed modifications greatly reduce the overall noise level, and diminish the fluctuation before $t=200$ for the high-spin case. The curves of $\chi$ with the modified BSSN formulation show less decay in each case. For the lower spin case, $\chi_{0}=0.53$, the curve for the modified BSSN formulation (red solid lines) is basically the same as the one for the traditional BSSN formulation (dashed lines). As the spin becomes higher, the loss of the angular momentum is more severe. For the $\chi_{0}=0.9$ case, the dimensionless spin drops more than $11 \%$ to 0.7986 at $t=1200$ in the traditional BSSN formulation, compared to the modified one in which $\chi$ drops less than 5\%. This result indicates that the modified BSSN formulation is capable to conserve the angular momentum better than the traditional BSSN, especially for the high spin cases, i.e., $\chi_{0}>0.75$.

The time evolution of the dimensionless spin is shown in the left panel of Fig. 3 for the near-extreme single black hole with $\chi_{0}=0.923$, which is nearly the maximal value that the conformally flat Bowen-York data can achieve. The dimensionless spin drops about $15 \%$ to 0.79 at $t=$ 1000 in the traditional BSSN formulation, compared to the modified case in which the change of $\chi$ is less than $5 \%$. It shows that the modified BSSN formulation is more effective in conserving the angular momentum over the traditional BSSN formulation, even in the fast-spinning SBH case.

It is interesting to study the different effect on the sim- 

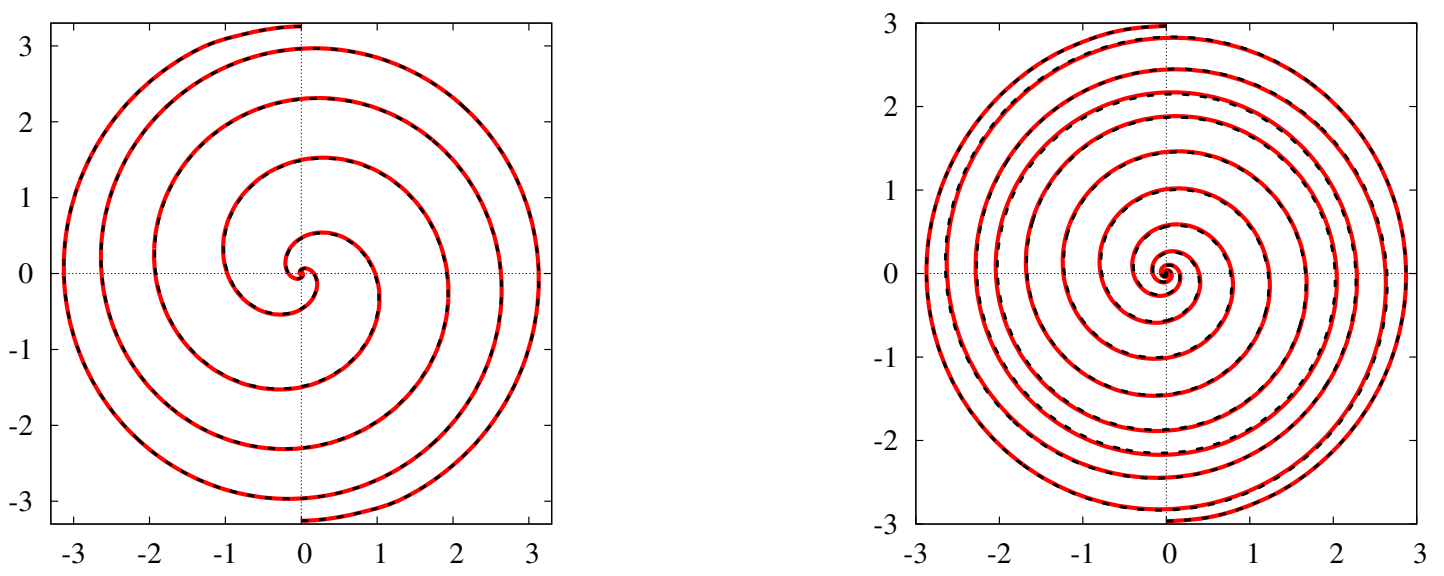

FIG. 4: The puncture trajectory of the binary black hole evolution with initial $\chi_{i}=0$ (left) and $\chi_{i}=0.9$ (right). The comparisons between the result with the traditional BSSN formulation (dashed line) and that with the modified BSSN formulation (solid red line) are shown.
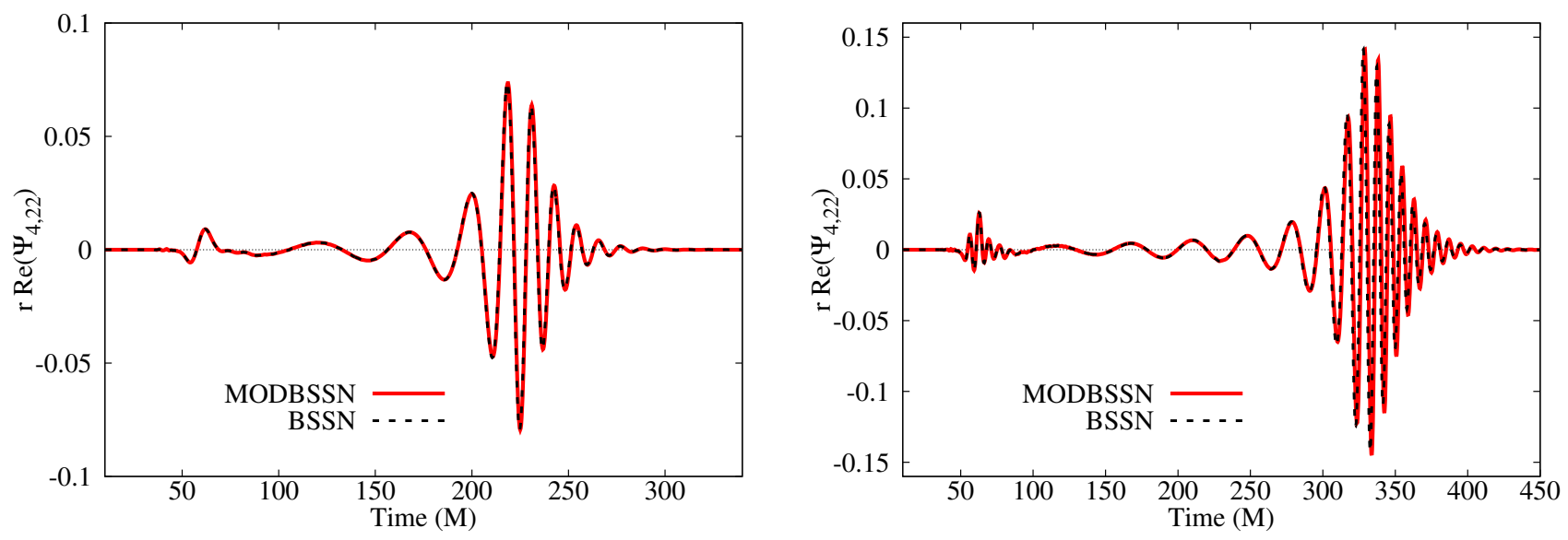

FIG. 5: The $\ell=2, m=2$ mode of the Newman-Penrose scalar $\Psi_{4}$ calculated at $R=50 M$, with initial $\chi_{i}=0$ (left) and $\chi_{i}=0.9$ (right). This mode is the major component of gravitational radiation during the merger in the binary black hole case with spin parallel to the orbital angular momentum. Only the real part of $\Psi_{4}$ is shown here. These two waveforms are almost identical for the traditional BSSN formulation (dashed line, marked as BSSN) and the modified one(solid red line, marked as MODBSSN). The binary composed of higher-spin hole spend more inspiral period due to the spin hang-up effect.

ulations between the $5^{\text {th }}$-order KO dissipation and Modification M3. From a naive observation on Fig. 2 and the left panel of Fig. 3. we found that the KO method is good at eliminating relatively higher frequency numerical noise. It can be seen that the result in right panel of Fig. 2 for the $\chi_{0}=0.9$ case with the traditional BSSN formulation (dashed line) is smoother than its counterpart with the modified BSSN formulation (red solid line), despite the spin drop in the former one. In contrary, the lower frequency numerical noise appearing in the traditional BSSN formulation is diminished significantly with the modified BSSN formulation. This can also be seen in the left panel of Fig. 2 wherein the two major fluctuations at $t \approx 580$ and $t \approx 1150$ with the traditional BSSN formulation (dashed lines) disappear with the modified BSSN formulation. It also can be seen in the right panel wherein the severe fluctuations before $t \approx 150$ with the traditional BSSN formulation is effectively suppressed with the modified BSSN formulation. It has similar behavior in the left panel of Fig. 3 .

To understand this phenomenon better, a Fourier analysis method is applied to the $\chi_{0}=0.923$ single black hole case. In the right panel of Fig. 3, we show the corresponding power spectrum of the data in the left panel. From this power spectrum, we can see that the KO method in the traditioinal BSSN formulation only dissipates some high-frequency $(f \sim 0.34-0.43)$ noise better. For the noise in the most other frequency, the M3 method in the modified BSSN formulation is much more efficient in dissipation. This difference results in the different behavior 

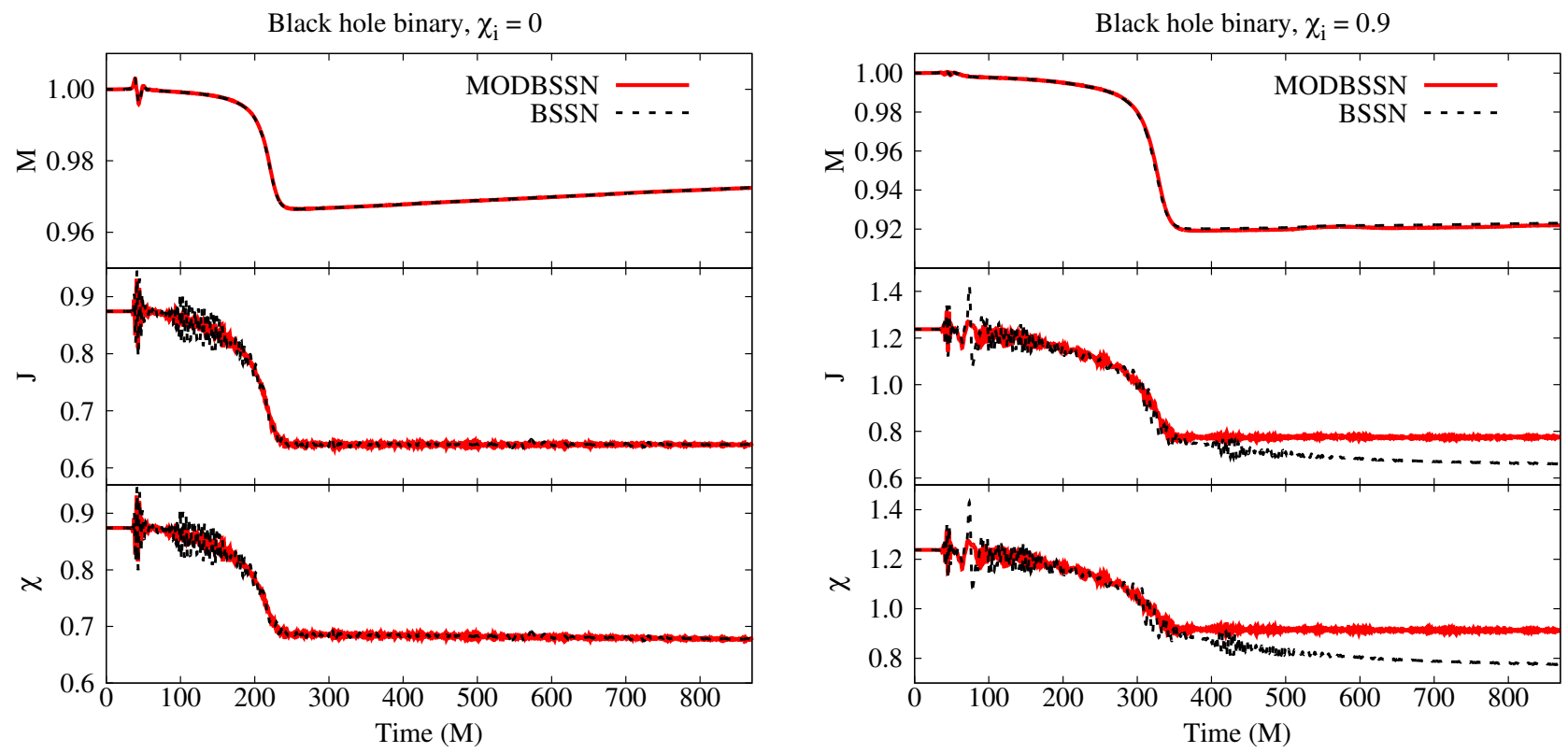

FIG. 6: ADM mass $M$, angular momentum $J$, and dimensionless spin parameter $\chi$ as functions of time for the initial $\chi_{i}=0$ (left column) and the $\chi_{i}=0.9$ (right column) in the binary black hole evolutions. The modified BSSN formulation (solid red line, marked as MODBSSN) has better control than the traditional BSSN(dashed line) on the early noise level and the spin drop after merger for the higher spin case.

in the left panel as we can see. The line for traditional BSSN formulation has larger amplitude oscillations with the intermediate frequency. The similar results can be seen in Fig. 2. The above result indicates that the KO dissipation and M3 suppress the numerical noise in different frequency ranges. It is noted that usage of M3 does not introduce any artificial dissipation and thus the field equation of $\tilde{A}_{i j}$ is analytically equivalent to the original.

\section{B. Binary black hole tests}

In this subsection, we apply our modifications to the equal-mass black hole binary. Each black hole in the binary has the spin aligned with the orbital angular momentum and the dimensionless spin parameter $\chi_{i}=0.9$ initially. As the reference, we also run an equal-mass $\mathrm{BBH}$ with $\chi_{i}=0$ for each black hole. The initial parameters for each hole as the input of the TwoPuncture solver are listed in Table \.

TABLE I: Parameters for the binary black hole puncture initial data

\begin{tabular}{ccc}
\hline \hline$\chi_{i}$ & 0 & 0.9 \\
\hline bare mass & 0.483 & 0.1764 \\
$\vec{r}$ & $\pm 3.257 \hat{\mathbf{y}}$ & $\pm 2.966 \hat{\mathbf{y}}$ \\
$\vec{p}$ & $\mp 0.133 \hat{\mathbf{x}}$ & $\mp 0.12616 \hat{\mathbf{x}}$ \\
$\vec{s}$ & 0 & $0.225 \hat{\mathbf{z}}$ \\
\hline
\end{tabular}

Firstly we would like to check if our modifications give any changes in these well-tested $\mathrm{BBH}$ cases, compared to the traditional BSSN formulation. Figures 4 and 5 show the almost identical puncture trajectories and the $\ell=2, m=2$ mode of the Newman-Penrose scalar $\Psi_{4}$ at $R=50 M$ for the cases of $\chi_{i}=0$ and $\chi_{i}=0.9$. The $\ell=2, m=2$ mode gives the major component of gravitational radiation during the merger in the case of the binary black hole with spin parallel to the orbital angular momentum. Here we only show the real part of $\Psi_{4}$. This result is expected in developing new modifications since all of these formulations are analytically equivalent to Einstein's field equations and should give same physics.

The ADM mass $M$, the angular momentum $J$, and the dimensionless spin parameter $\chi$ are shown in Fig. 6 for the BBH cases with the initial dimensionless spin $\chi_{i}=0$ (left) and $\chi_{i}=0.9$ (right). After the merger at $t=250$ in the $\chi_{i}=0 \mathrm{BBH}$ case, $M$ and $J$ decrease by $4 \%$ and $27 \%$ respectively due to the gravitational radiation. And the dimensionless spin parameter after the merger is $\chi=0.68$ at $t=250$ to $\chi=0.67$ at $t>800$. Thus $\chi$ decreases less than $2 \%$ after $t=250$ until the end of simulation. It also shows that the modified and traditional BSSN formulations give the same result (in the left panel) in the initially slowly spinning BBH case. In the $\chi_{i}=0.9$ case, after the merger at $t=350$, the gravitational radiation decreases the value of $M$ and $J$ by $8 \%$ and $39 \%$ respectively. As shown in the right panel for the $\chi_{i}=0.9$ case, $\chi$ in the traditional BSSN formulation decreases more than $10 \%$ from $t=350$ to $800(\chi \approx 0.75$ in our extended run for $t>1900$ ). This result from the traditional BSSN formulation is consistent with the discovery in [36] in which the final $J$ will decay considerably for $\chi_{i} \geq 0.75$. For the $\chi_{i}=0.9$ case with the modified BSSN formulation, $\chi$ de- 
creases only by $1 \%$ from $t=350$ to 800 . The decrease is still less than $2 \%$ in the extended run for $t>1900$. The results in the $\mathrm{BBH}$ cases, combined with that in the $\mathrm{SBH}$ cases, indicate clearly that our modifications handle the highly spinning black holes much better than the traditional BSSN formulation, while yielding the same results as in traditional BSSN formulation in the slow spinning black hole cases.

\section{Numerical Convergence}

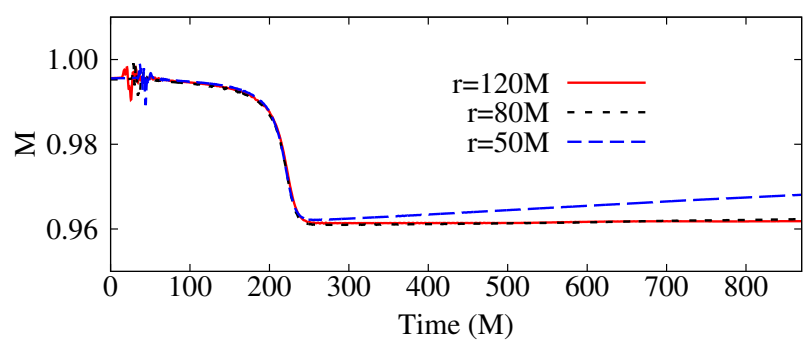

FIG. 7: Effect of the extraction radius on the ADM mass integration. The plot corresponds to the spinless binary black hole case. The r's in the legend are the extraction radii used in this case.

For both the gravitational wave extraction and the calculation of the global quantities, they are numerically integrated on the sphere of radius $r=50 M$. This finite radius for integration could affect the accuracy of the amplitude of the gravitational waveform $\Psi_{4}$. However, the effect from the integration sphere of finite radius should be roughly the same with either the traditional BSSN formulation or the modified one on any case. Since we are only concerned about the relative difference between these two formulations, the effect from the extraction radius becomes unimportant. For the global quantities, e.g., the ADM mass and the angular momentum, the integration sphere of finite radius, e.g., $r=50 M$, may result in some artificial drift as shown in Fig. 6. Nevertheless, when an integration sphere with larger radius is applied to the case, such kind of drift diminishes. Here we use the spinless binary black hole case as an example in Fig. 7 for illustration. According to Fig. 7 the result with $r=50 M$ are basically same as the ones with $r=80 M$ and $r=120 M$ during the merger phase. The drift in the case with $r=50 M$ only shows during the ringdown stage. And the drift can be easily diminished with larger radii, e.g., $r=120 M$ in Fig. 7. However, in order to compare our result with the one in [36] closely, in this work we still take the radius $r=50 M$, same as used in 36.

The physical boundary used in simulations may also affect the gravitational wave and the calculation of the global quantities. In order to investigate such possi- ble effects, we have tested the simulations with farther boundaries. And our results show that the effect from the boundary condition is ignorable in the current work.

According to the arguments in Sec. II analytically we do not expect Modification M3 to affect the convergence of the system, and we do expect that Modification M1 definitely affects the system's numerical convergent behavior due to the switch character of the step function in Eq. (2). Here we would like to both check the convergence order of the system with the modified BSSN formulation and verify these arguments numerically.

Firstly, we show the system's convergence with our modifications in the left-side panels of Fig. 8, compared with the one with the traditional BSSN formulation. Here we use the $\chi_{i}=0$ binary black hole case as an example for demonstration. In the two plots we study the convergence of the phase and the amplitude for the gravitational wave respectively. According to the plots, the traditional BSSN formulation results in overall $3.3^{\text {th }}$-order convergence for the system in both the phase and amplitude of $\Psi_{4}$, which is roughly consistent with the ideal convergence of fourth-order with the numerical method used in this work. On the other hand, it shows in the panels that the modified one results in only first-order convergence for the system. Since we already expect that some of our modifications will affect the convergence of the system, the result is understandable, although the order of convergence is still considered low. And we can see from Fig. 8 that the numerical error with the modified BSSN formulation is much smaller than the one with the traditional BSSN formulation, especially in the lower resolutions. This merit for the modified BSSN formulation could compensate for its disadvantage of having lower order convergence. And the convergence behavior showed in Fig. 8 is general for all the cases we have done in this work.

Secondly, we would like to confirm the theoretical analysis that it is Modification M1, not M3, in the modified BSSN formulation which majorly affects the convergence order. By using again the $\chi_{i}=0$ binary black hole case as an example, we show the result in the right-side panels of Fig. 8. When we apply the traditional BSSN formulation $+\mathrm{M} 1$ to the case, the resulted convergence order is first-order, which is roughly the same as the convergence order for the case with the whole modified BSSN formulation. Meanwhile, when we apply the traditional BSSN formulation + M3 to the case, the resulted convergence order is $2.5^{\text {th }}$-order, which is a little lower than the case with the pure traditional BSSN formulation, but quite higher than the one with the modified one.. The result tells that M1 is the key modification which affects majorly the convergence behavior of the system, as we expected. However, it also shows that M3 lowers minorly the convergence order of the system. This indicates that the convergence order of M3 might not be numerically as good as the expectation from our analytical argument [40]. In conclusion, the cases with our modifications have first-order convergence, which is lower than the one with 

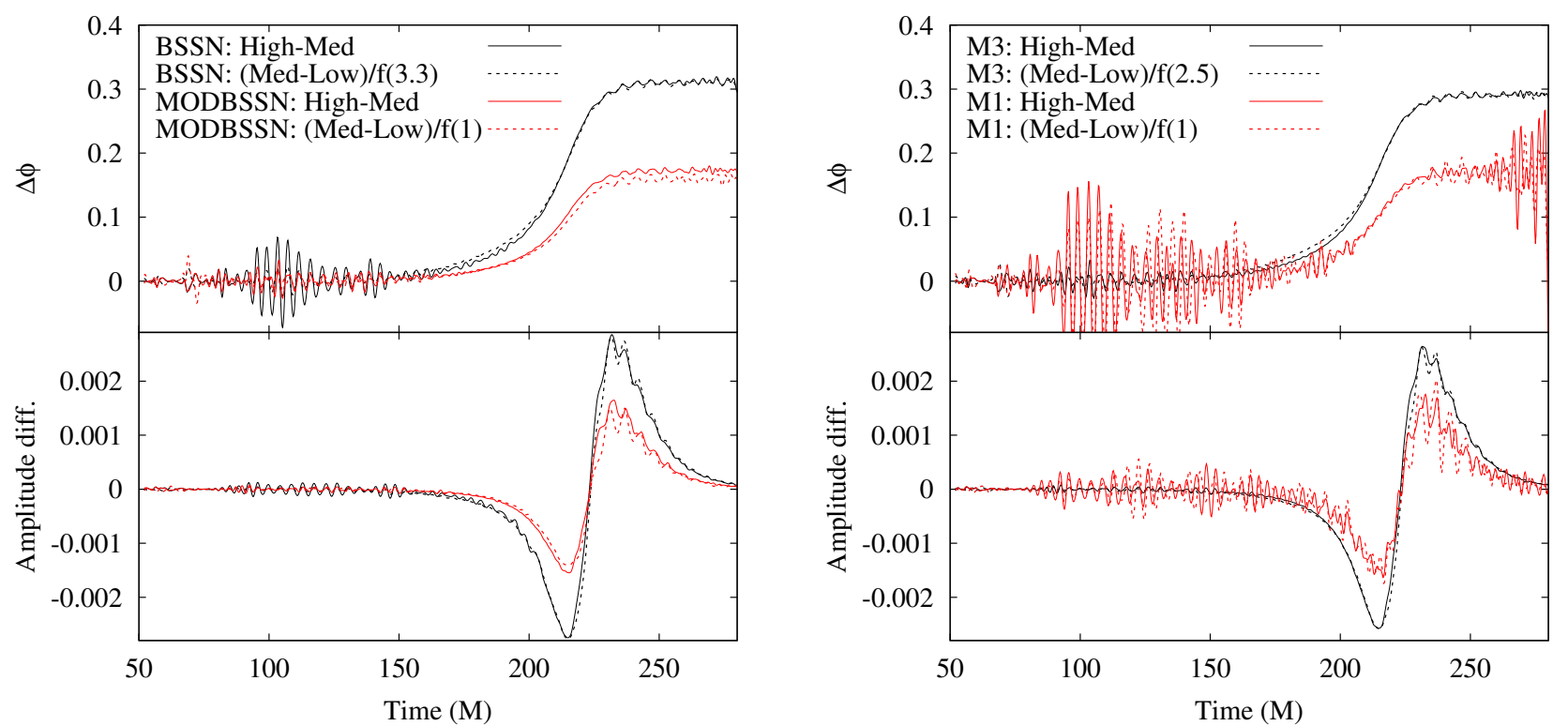

FIG. 8: Convergence of gravitational wave for the $\chi_{i}=0$ binary black hole case. The left-side panels show the phase differences and the amplitude differences of $\Psi_{4}$ respectively between the high and medium resolutions (solid line), and the medium and low resolutions (dotted line), with both the traditional BSSN formulation (BSSN, black line) and the modified one (MODBSSN, red line). Here we use $f(p)$ to denote the factor of order $p$. The right-side panels show the phase differences and the amplitude differences of $\Psi_{4}$ respectively between the high and medium resolutions (solid line), and the medium and low resolutions (dotted line), with the traditional BSSN formulation+Modification M3 only (M3, black line) and the traditional one+Modification M1 only (M1, red line).

the traditional BSSN formulation, but our modifications give more accurate result than the traditional BSSN formulation at a given resolution. And Modification M1 is the key factor in our modifications to affect the convergence behavior.

\section{DISCUSSION AND SUMMARY}

In this work, we applied our modifications of the BSSN formulation to study the total angular momentum conservation issue in black hole evolutions with the standard Bowen-York puncture initial data. We found that the non-negligible loss of angular momentum for highly spinning black holes mentioned in [36] can be greatly cured with our modifications. The improvements are obvious for near-extreme cases, as in the SBH case shown in Fig.2 and Fig. 3 and the BBH case in Fig. 6] It has also been shown in the previous section that the modified BSSN formulation does not introduce any unphysical effects. Improving the conservation of the angular momentum usually leads to certain improvement on the accuracy of the results in black hole evolutions. Therefore we expect that our modifications will provide better performance in black hole evolution simulations than the traditional BSSN scheme.

Modification M1 is the most important to the conservation of the angular momentum since the field equation of the conformal connection function $\tilde{\Gamma}^{i}$ is closely related to the (angular) momentum vector. We find that Eq. (2) and setting $\xi=1$ gives quite robust and stable runs. But due to the switch character of M1 and the possible sign fluctuation of the numerical value of its argument when the argument is close to zero, the step function is sensitive to the resolution used in simulations. So this modification affects majorly the numerical convergence order of the modified BSSN formulation. M2 is able to enhance the hyperbolicity of the system, especially for the evolution of $\tilde{\Gamma}^{i}$. However, its mechanism and the optimal choice of $\sigma$ need further investigations.

Instead of the $\mathrm{KO}$ dissipation method used in the traditional BSSN formulation, Modification M3 is used in the modified BSSN formulation in this work. We can see in Sec. III that M3 is able to diminish effectively some intermediate frequency noise with larger amplitude; while the $\mathrm{KO}$ dissipation is good at eliminating the higher frequency numerical noise. To some extent, Modification M3 is complementary to the $\mathrm{KO}$ method in dissipating numerical error. However, the advantage of M3 is that it comes from the derivative of the momentum constraint. Thus, applying M3 to the BSSN formulation is always legitimate and safe as long as the momentum constraint holds. In contrast, the application of the KO method is not always safe since it is an artificial addition to the field equation, although it is convenient and effective in numerical relativity. It is possible that usage of the $\mathrm{KO}$ method leads to deviations of the numerical result from 
the solution hypersurface, especially when the result is sensitive to the initial data. It will be a good idea to use both Modification M3 and the KO method in dissipating the numerical error in the future simulations.

As mentioned at the beginning of Sec. III in order to enforce the algebraic constraints of the unimodular determinant of $\tilde{\gamma}_{i j}$, and of $\tilde{A}_{i j}$ being traceless, the numerical values of $\tilde{\gamma}_{i j}$ and $\tilde{A}_{i j}$ are replaced with $\tilde{\gamma}_{i j} \rightarrow \tilde{\gamma}^{-1 / 3} \tilde{\gamma}_{i j}$, $\tilde{A}_{i j} \rightarrow \tilde{A}_{\langle i j\rangle}$ after every time step in the traditional BSSN formulation. However, the modification

$$
\begin{aligned}
& \tilde{\gamma}_{z z} \rightarrow \frac{1+\tilde{\gamma}_{y y} \tilde{\gamma}_{x z}^{2}-2 \tilde{\gamma}_{x y} \tilde{\gamma}_{y z} \tilde{\gamma}_{x z}+\tilde{\gamma}_{x x} \tilde{\gamma}_{y z}^{2}}{\tilde{\gamma}_{x x} \tilde{\gamma}_{y y}-\tilde{\gamma}_{x y}^{2}}, \\
& \tilde{A}_{y y} \rightarrow \frac{\tilde{A}_{x}^{x}+\tilde{A}_{z}^{z}+\tilde{A}_{x y} \tilde{\gamma}^{x y}+\tilde{A}_{y z} \tilde{\gamma}^{y z}}{\tilde{\gamma}^{y y}},
\end{aligned}
$$

is employed instead in 1] to enforce the two constraints. The results in [1] have shown that this modification gains better stability compared to the traditional BSSN formulation. Differing with [1], in this work we use the traditional recipe of $\tilde{\gamma}_{i j} \rightarrow \tilde{\gamma}^{-1 / 3} \tilde{\gamma}_{i j}, \tilde{A}_{i j} \rightarrow \tilde{A}_{\langle i j\rangle}$ instead of applying the modification Eqs. (10) \& (11). This is because the denominators in Eqs. (1011) can be very small near the singularities and thus the numerical values of the replaced $\tilde{\gamma}_{z z}$ and $\tilde{A}_{y y}$ can have unexpected fluctuation which can crash the code. However, this modification can still be applied to the BSSN formulation if there is no singularity or if an excision method is used in the black hole evolution simulations.

In the modifications, we introduce some terms related to the spacial resolution used in the numerical simulation. These terms reduce the convergence order from fourthorder to first-order in our implementation. But compared with the traditional BSSN formulation, the numerical error resulted in the modified BSSN formulation is still obviously smaller than the one from the traditional BSSN formulation with a reasonably fine resolution.

In this work, we demonstrated the simulations with the modified BSSN formulation in which the angular momentum conservation is better than in the traditional BSSN formulation. Thus this modified BSSN formulation should improve the accuracy in the punctured black hole evolutions. Our modifications are imposed on the field equations of the physical variables $\tilde{\gamma}_{i j}, \tilde{A}_{i j}$ and $\tilde{\Gamma}^{i}$, instead of the gauge variables $\alpha$ and $\beta^{i}$. Therefore, we expect that the modified BSSN formulation can be applied generally to various scenarios to give improved results in numerical relativity.

\section{Acknowledgments}

This work was supported in part by the National Science Council under Grants No. NSC102-2112-M-006-014MY2, and by the Headquarters of University Advancement at the National Cheng Kung University, which is sponsored by the Ministry of Education, Taiwan, ROC. Z. Cao was supported by the NSFC (No. 11375260). We are grateful to the National Center for High-performance Computing for the use of their computer time and facilities. We are also grateful to the Academia Sinica Computing Center for providing computing resource; and to Peter Diener for helpful discussions. HJY thanks the hospitality of ASIAA for hosting his visit from July 2014 to February 2015.
[1] H.-J. Yo, C.-Y. Lin, and Z. Cao, Phys. Rev. D 86, 064027 (2012), URL http://link.aps.org/doi/10.1103/PhysRevD.86.064027

[2] F. Pretorius, Physical review letters 95, 121101 (2005).

[3] M. Campanelli, C. O. Lousto, P. Marronetti, and Y. Zlochower, Physical review letters 96, 111101 (2006).

[4] J. G. Baker, J. Centrella, D.-I. Choi, M. Koppitz, and J. van Meter, Physical review letters 96, 111102 (2006).

[5] Y. Pan, A. Buonanno, L. T. Buchman, T. Chu, L. E. Kidder, H. P. Pfeiffer, and M. A. Scheel, Phys. Rev. D 81, 084041 (2010), URL http://link.aps.org/doi/10.1103/PhysRevD.81.084041

[6] M. Campanelli, C. O. Lousto, Y. Zlochower, and D. Merritt, Physical Review Letters 98, 231102 (2007).

[7] C. O. Lousto and Y. Zlochower, Physical Review D 77, 044028 (2008)

[8] Y. Zlochower, M. Campanelli, and C. O. Lousto, Classical and Quantum Gravity 28, 114015 (2011).

[9] C. O. Lousto and Y. Zlochower, Physical Review D 83, 024003 (2011).

[10] C. O. Lousto, Y. Zlochower, M. Dotti, and M. Volonteri, Physical Review D 85, 084015 (2012).
[11] J. S. Read, C. Markakis, M. Shibata, K. Uryū, J. D. Creighton, and J. L. Friedman, Physical Review D 79, 124033 (2009).

[12] S. Bernuzzi, M. Thierfelder, and B. Brügmann, Phys. Rev. D 85, 104030 (2012), URL http://link.aps.org/doi/10.1103/PhysRevD.85.104030

[13] S. Bernuzzi, A. Nagar, M. Thierfelder, and B. Brügmann, Phys. Rev. D 86, 044030 (2012), URL http://link.aps.org/doi/10.1103/PhysRevD.86.044030

[14] K. Hotokezaka, K. Kyutoku, and M. Shibata, Phys. Rev. D 87, 044001 (2013), URL http://link.aps.org/doi/10.1103/PhysRevD.87.044001

[15] J. C. McKinney and R. D. Blandford, Monthly Notices of the Royal Astronomical Society: Letters 394, L126 (2009).

[16] C. Palenzuela, L. Lehner, and S. L. Liebling, Science 329, 927 (2010).

[17] K. Kiuchi, Y. Sekiguchi, M. Shibata, and K. Taniguchi, Physical review letters 104, 141101 (2010).

[18] Y. Sekiguchi and M. Shibata, The Astrophysical Journal 737, 6 (2011).

[19] M. Shibata and T. Nakamura, Physical Review D 52, 
5428 (1995).

[20] T. W. Baumgarte and S. L. Shapiro, Physical Review D 59, 024007 (1998).

[21] M. Alcubierre and B. Brügmann, Physical Review D 63, 104006 (2001).

[22] H.-J. Yo, T. W. Baumgarte, and S. L. Shapiro, Physical Review D 66, 084026 (2002).

[23] G. Yoneda and H. Shinkai, Physical Review D 66, 124003 (2002).

[24] K. Kiuchi and H.-a. Shinkai, Physical Review D 77, 044010 (2008).

[25] T. Tsuchiya, G. Yoneda, and H.-a. Shinkai, Physical Review D 85, 044018 (2012).

[26] J. D. Brown, P. Diener, S. E. Field, J. S. Hesthaven, F. Herrmann, A. H. Mroué, O. Sarbach, E. Schnetter, M. Tiglio, and M. Wagman, Physical Review D 85, 084004 (2012).

[27] L. Lindblom, M. A. Scheel, L. E. Kidder, R. Owen, and O. Rinne, Classical and Quantum Gravity 23, S447 (2006).

[28] S. Bernuzzi and D. Hilditch, Physical Review D 81, 084003 (2010)

[29] D. Alic, C. Bona-Casas, C. Bona, L. Rezzolla, and C. Palenzuela, Physical Review D 85, 064040 (2012).

[30] D. Alic, W. Kastaun, and L. Rezzolla, Phys. Rev. D 88, 064049 (2013), URL http://link.aps.org/doi/10.1103/PhysRevD.88.064049

[31] A. Weyhausen, S. Bernuzzi, and D. Hilditch, Physical Review D 85, 024038 (2012).
[32] D. Hilditch, S. Bernuzzi, M. Thierfelder, Z. Cao, W. Tichy, and B. Brügmann, Physical Review D 88, 084057 (2013).

[33] P. Laguna and D. Shoemaker, Classical and Quantum Gravity 19, 3679 (2002).

[34] Z. Cao, H.-J. Yo, and J.-P. Yu, Physical Review D 78, 124011 (2008)

[35] Z. B. Etienne, J. G. Baker, V. Paschalidis, B. J. Kelly, and S. L. Shapiro, arXiv preprint arXiv:1404.6523 (2014).

[36] P. Marronetti, W. Tichy, B. Bruegmann, J. Gonzalez, and U. Sperhake, Physical Review D 77, 064010 (2008).

[37] D. A. Hemberger, G. Lovelace, T. J. Loredo, L. E. Kidder, M. A. Scheel, B. Szilágyi, N. W. Taylor, and S. A. Teukolsky, Phys. Rev. D 88, 064014 (2013), URL http://link.aps.org/doi/10.1103/PhysRevD.88.064014

[38] P. Galaviz, B. Brügmann, and Z. Cao, Phys. Rev. D 82, 024005 (2010), URL http://link.aps.org/doi/10.1103/PhysRevD.82.024005

[39] S. Dain, C. O. Lousto, and Y. Zlochower, Physical Review D 78, 024039 (2008).

[40] It is quite possible that the numerical convergence order with Modification M3 is lower than the one with pure traditional BSSN formulation due to some numerical residual from the finite-differencing in M3, although its convergence order is designed to be higher than analytically. However, in the modified BSSN formulation, its convergence order turns out to be irrelevant as long as its order is higher than the convergence order of M1. 